\title{
Health anxiety and Internet use: A thematic analysis
}

\author{
Karmpaul Singh, John R. E. Fox, Richard J. Brown \\ University of Manchester, Manchester, United Kingdom
}

\begin{abstract}
The current study aimed to obtain health anxious students' perspectives on their reasons for using the Internet to obtain health information, and the nature and effects of such usage. Data were gathered using semi-structured interviews with 20 postgraduate and undergraduate students identified as highly health anxious, and were examined using thematic analysis. Results suggested that themes were organized by different stages of the search process. Reasons for searching included curiosity, anxiety/worry about undiagnosed symptoms, and remedy-seeking. Both positive (e.g. reassurance) and negative (e.g. uncertainty) outcomes were reported. Findings from the current study suggest that the Internet constitutes an important resource for obtaining health information by health anxious individuals, with the potential to both reduce and exacerbate health anxiety.
\end{abstract}

Keywords: Hypochondria; cyberchondria; qualitative; internet; health anxiety

\section{Introduction}

Evidence suggests that up to 50\% of Europeans (European Commission, 2013) and 72\% of Americans (Fox, 2013) used the Internet to search for health information in 2013 alone, a figure that has likely risen since. Despite the apparent benefits of being able to access online health resources, a number of commentators have expressed concerns about the potentially anxiety-provoking effects of online medical information (e.g., Muse, McManus, Leung, Meghreblian, \& Williams, 2012; Singh \& Brown, 2014, 2015; Starcevic \& Berle, 2013). Evidence indicates that Internet use for health purposes is higher in health anxious people (Muse et al., 2012; Singh \& Brown, 2014, 2015), with increased distress and doctor utilization being comparatively common responses to Internet use in these individuals (Baumgartner \& Hartmann, 2011; Eastin \& Guinsler, 2006; Muse et al., 2012; Singh \& Brown, 2014). Indeed, even those with lower state health anxiety are at risk of increased feelings of anxiety post health related Internet search (Singh \& Brown, 2015). Furthermore, health anxious individuals were also more likely to endorse items suggesting possible addiction to health related Internet use in one recent study (Singh \& Brown, 2014), suggesting that usage could become problematic in its own right.

According to the cognitive-behavioural model (CBT; Warwick \& Salkovskis, 1990), an episode of health anxiety can occur when dysfunctional health related beliefs (e.g. "dizziness is a sign of brain cancer") are activated by internal (i.e. physical symptoms) or external (i.e. media stories, family illness) stimuli, causing them to be misinterpreted as evidence of a serious health threat. Once activated, cognitive (e.g. catastrophic misinterpretation), behavioural (e.g. reassurance seeking), physiological (e.g. increase autonomic arousal), and affective (e.g. depression) factors are thought to maintain anxiety and preoccupation with the idea that one currently has (or will develop) a serious illness. Common illness cognitions in health anxious individuals include erroneous beliefs about the likelihood of currently having or acquiring a serious illness (Abramowitz, Olatunji, \& Deacon, 2007; Marcus \& Church, 2003) and beliefs concerning the dangerousness and intolerability of 
uncertainty (Deacon \& Abramowitz, 2008). Intolerability of uncertainty may also prompt health related Internet use in health anxious individuals (Fergus, 2013) and may cause distress with this type of searching (Fergus, 2015; Norr, Albanese, Oglesby, Allan, \& Schmidt, 2015). In relation to behavioural maintenance factors, there is evidence (Conroy, Smyth, Siriwardena, \& Fernandes, 1999; Hadjistavropoulos, Craig, \& Hadjistavropoulos, 1998; Warwick \& Marks, 1988) that health anxious individuals will seek reassurance from sources such as the doctor and/or friends and family. Although originally formulated in more general terms, these factors may also pertain to health related Internet use (Singh \& Brown, 2014) in an apparent attempt to reduce anxiety and uncertainty about their health status (Singh \& Brown, 2015; Starcevic \& Berle, 2013).

Although seeking reassurance is a normal and appropriate response to health threats, its anxiety-reducing effects are only short-lived in health anxious individuals and can negatively reinforce health preoccupation (Starcevic \& Berle, 2013; Wells, 1997). Indeed, reassurance seeking may be a particularly important reason for such online health related searching, and a behavior that poses a particular risk to health anxious individuals (Starcevic \& Berle, 2013). Research supports this idea, where health anxious individuals were found to seek reassurance by often examining the relevance of online health information to one's own symptoms (Singh \& Brown, 2015).

Furthermore, seeking to "diagnose" one's symptoms online may increase the likelihood of noticing and misinterpreting benign stimuli as evidence for disease; triggering anxiety and illness preoccupation (Singh \& Brown, 2015; Warwick \& Salkovskis, 1990). Though health related Internet usage (in non-health anxious samples) has been studied by researchers in the past (e.g. Eysenbach \& Kohler, 2002; Gray, Klien, Noyce, Sesselberg, \& Cantrill, 2005), CBT theory suggests health anxious individuals are more inclined to search online and experience negative effects, due to the abovementioned maintenance factors (Starcevic \& Berle, 2013; Wells, 1997). Therefore, those with health anxiety may differ in their online health related usage and behaviours from those who are not health anxious.

To our knowledge, however, no published study has directly examined why and how health anxious people use the Internet for health purposes from their own point of view, including how they interact with and consider online health information, and whether their health anxiety has any bearing on their Internet use (or vice versa). Studies conducted in this area thus far have been primarily quantitative and theory-based (e.g. Singh \& Brown, $2014,2015)$. As such, these studies are constrained to theory driven areas of importance. However, it is essential to understand how health anxious users perceive the Internet for health purposes themselves, as this may lead to valuable information that can extend theory to areas (i.e. thoughts and behaviors) not yet considered in an online setting.

The current study was designed to explore how health anxious individuals perceive their health related Internet use, and to generate ideas about how the Internet might contribute to health anxiety to inform future theory/research. To do this we opted to utilize thematic analysis due to its flexibility in acquiring and understanding rich, qualitative data (Braun \& Clarke, 2006). As it was not our intention to develop a new theory with our findings (we were mainly pursuing to understand a health anxiety CBT model) we decided against using techniques such as Grounded Theory (Corbin \& Strauss, 2014, p.7). Relatedly, we opted against using Interpretive Phenomenological Analysis (IPA), because we were interested in drawing out general themes rather than describing each health anxious individual's idiosyncratic perceptions and experiences (Smith, 1996). Due to the infancy of this research area we felt that these themes would allow us to inform future research better than an IPA approach.

\section{Method}

\section{Participants}

A convenience sample of sixty-six undergraduate and postgraduate psychology students at the University of Manchester were recruited online using a voluntary credit-for-participation scheme. After providing informed consent, participants completed the Short Health Anxiety Inventory (SHAl; Salkovskis, Rimes, Warwick, \& Clark, 2002). The SHAl is an 18 item measure, with each item scored from 0-3. Total scores range from 0-54. There is disagreement as to what constitutes clinically significant health anxiety using the SHAl (Alberts, 
Hadjistavropoulos, Jones, \& Sharpe, 2013). However, scores greater or equal to 18 have been used previously for this purpose (Muse et al., 2012; Singh \& Brown, 2014, 2015), and is the recommended cut-off standard according to the UK National Health Service (NHS; NHS-IAPT, 2011). SHAI items were scored according to these criteria for the current study. The current study utilized a flexible sample size to achieve satisfactory data saturation within a confined time span. Thirty-five individuals scoring greater than or equal to 18 on the SHAI were invited to an interview to discuss their health related Internet use, with the first twenty volunteers taking part. All participants were recruited online specifically for their health related Internet use. As theoretical saturation was achieved after interviewing twenty participants, data collection was terminated at that point.

The demographics of the twenty interviewed participants were 7 males (35\%) and 13 females (65\%). Age range 18-32 years; mean age 24.60 years $(S D \pm 5.71)$. Fifteen of 20 participants identified themselves as Caucasian (75\%). The remainder (5) of the sample was 1 Oriental/Asian, 2 South Asian, 1 Mixed Race, and 1 did not specify. The mean SHAI score was 27.05 (SD \pm 5.03$)$.

\section{Research Context}

Semi-structured interviews were used to collect data for thematic analysis. To achieve this an interview schedule was produced using potential areas of importance based on prior findings in both research (Singh \& Brown, 2014; Muse et al., 2012) and theory (Creed \& Barsky, 2004; Starcevic \& Berle, 2013; Wells, 1997). Previous research and theory has highlighted several areas of importance relating to Internet use for health purposes including; heightened anxiety post-search (Muse et al., 2012; Singh \& Brown, 2014, 2015), post-search doctor utilization (Muse et al., 2012; Singh \& Brown, 2014), heightened reassurance seeking (which could include Internet use; Wells, 1997), and doctor distrust (prompting use of alternative resources to acquire information; Singh \& Brown, 2014; Starcevic, 2005).

Topics were chosen to cover a wide array of Internet-related health use to maximize data procurement and to explore areas as informed by participants. Earlier quantitative studies are hindered by their use of author-theory derived criteria of importance relative to health related searching. This qualitative study allows us to gather and understand areas of importance from the health anxious user's point of view. This both allows us to validate earlier quantitative findings (e.g. Muse et al., 2012; Singh \& Brown, 2014) and also inform future research to focus on areas health anxious participants believe are important to their health related online searching (see Appendix for interview guide).

Because CBT theory was partly used to produce topics to explore during interviews, there is always a risk that during data analysis, data could be selected/examined (consciously or unconsciously) to provide evidence for theory. Therefore, the analysis becomes theory driven rather than data driven. In the current study we maintain that only interview data was used to form themes and interpret results for analysis. Our aim was to use theory as a guide, not to purposefully provide qualitative evidence for theory. However, because we were using and were familiar with the CBT model of health anxiety, it is likely that theory was a likely influence on the interview process and interpretation of findings. To minimize this potential bias, prior to the commencement of the study six pilot interviews were conducted with health anxious participants, transcribed, and discussed with the second and third authors separately to establish question appropriateness. Data from the main study was also subjected to weekly meetings/discussions. Areas of the interview map aimed to address our research aims. For example, we attempted to determine why/how health anxious individuals used the Internet, and their experiences pertaining to health related Internet use; for this, areas such as motivation to search and how information was sought were included. Other areas of the map sought to supplement/clarify findings in these areas, or explore other possible areas of importance such as emotional impact etc. Areas specified on the interview map were kept broad in order to facilitate a conversational flow. All interviews were conducted at the University of Manchester by the first author.

\section{Interview Procedure}

The interviewer began by explaining the purpose of the interview and seeking consent to continue. Participants were encouraged to speak freely when answering and were told that there were no correct/incorrect answers. The interview began with broad questions about the extent of the individual's online health related searching. 


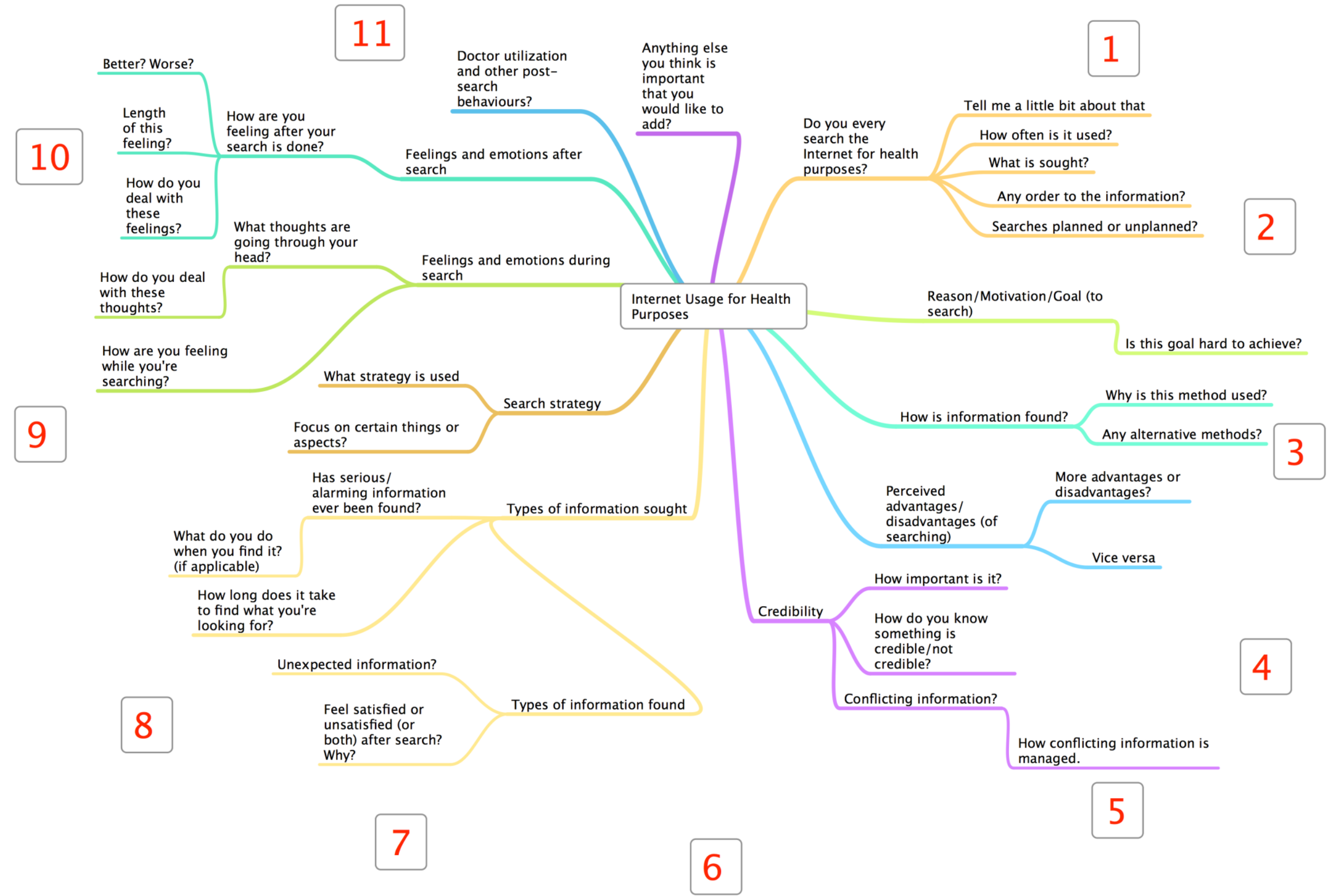

Figure 1. Interview Question Map (Clockwise Rotation Starting at "1 o'clock"). 
The interviewer then sought information in eleven general areas of interest, starting with planned questions followed by reflections/probes based on the participant's responses. When topics were felt to be fully explored, questioning moved to other areas. The order of questioning was loosely determined by the layout of the interview map (Figure 1), although deviations were allowed in order to maintain conversational flow (e.g., if the discussion naturally led onto another topic on the map). Interviews concluded when all map areas (including participant-determined areas) had been explored in detail, typically after 45-60 minutes. Interviews were recorded using QuickTime Player v.10.2, and transcribed using Microsoft Word 2010.

\section{Analysis}

Interviews were transcribed for thematic analysis using the approach of Braun and Clarke (2006). Transcription was completed between interviews to help determine data saturation. Data were coded line-by-line after all interviews had been conducted and transcribed, and were manually arranged into 52 broad themes (initially based on frequency, similarity of responses, and by interview-derived categories). Broad themes were then collapsed into core themes and then into sub-themes by eliminating redundancies, discarding minor/irrelevant themes and merging closely related major themes. These aimed to elucidate the potential relations between health related Internet searching and health anxiety. Core/sub-themes were typically only derived if at least half of the sample ( $\geq 10$ participants) spoke about the topic when describing their health related Internet use. Topics mentioned by $60-80 \%$ of the sample (13-16 participants) are described as "the majority" of participants in the current text. "Extremely common" is used to describe topics mentioned by 19 or all 20 participants.

Frequencies were used to highlight areas we felt were important for theme development and to clarify data presentation. We did not adopt a purely quantitative approach to classifying interview data into themes. In some cases, fewer than half of our sample mentioned an area/item (e.g. "assuming credibility" was derived from six participants), but were included because they seemed to add to our understanding of health related Internet use. We referred to these concepts as pertaining to "a minority" of participants.

\section{Findings}

The simplest way of organizing the results was to divide the data chronologically into core themes pertaining to different stages of the search process (i.e. before [predisposition to search/proximal pre-search], throughout [during search] or after searching the Internet [post-search]). Within these core themes, several sub-themes were identified. Figure 2 provides a simplified representation of these inter/intra-theme relationships, organized within this chronological framework. These are described and interpreted below.

\section{Predisposition to Search}

Data suggested a number of predisposing characteristics and processes that seemed to influence participants' subsequent decisions to search for health information online.

Uncertainty. Uncertainty related to the probability and likelihood of having a serious illness was present in the majority of our sample, and was a major contributor to assessing the need to search online in the proximal presearch stage. Uncertainty about unknown health issues primed illness thoughts and metacognitions (see below), heightening anxiety and worry in some cases. When uncertainty related to a currently experienced symptom, catastrophic thinking (i.e., initial triggering "what if..." thoughts regarding perceived illness seriousness) was a common outcome, which in some cases (e.g. when the issue was unfamiliar) led to worry and anxiety, and eventually if perceived to be serious enough, to a feeling of (i.e. non-clinical) "panic" in the proximal pre-search stage (with a decision to initiate an online health search rapidly; intolerance of uncertainty).

"...You think about all the different possibilities; it can be so many different things. I do feel anxious; it does go through my head first that it's something more serious..." (Participant 10) 


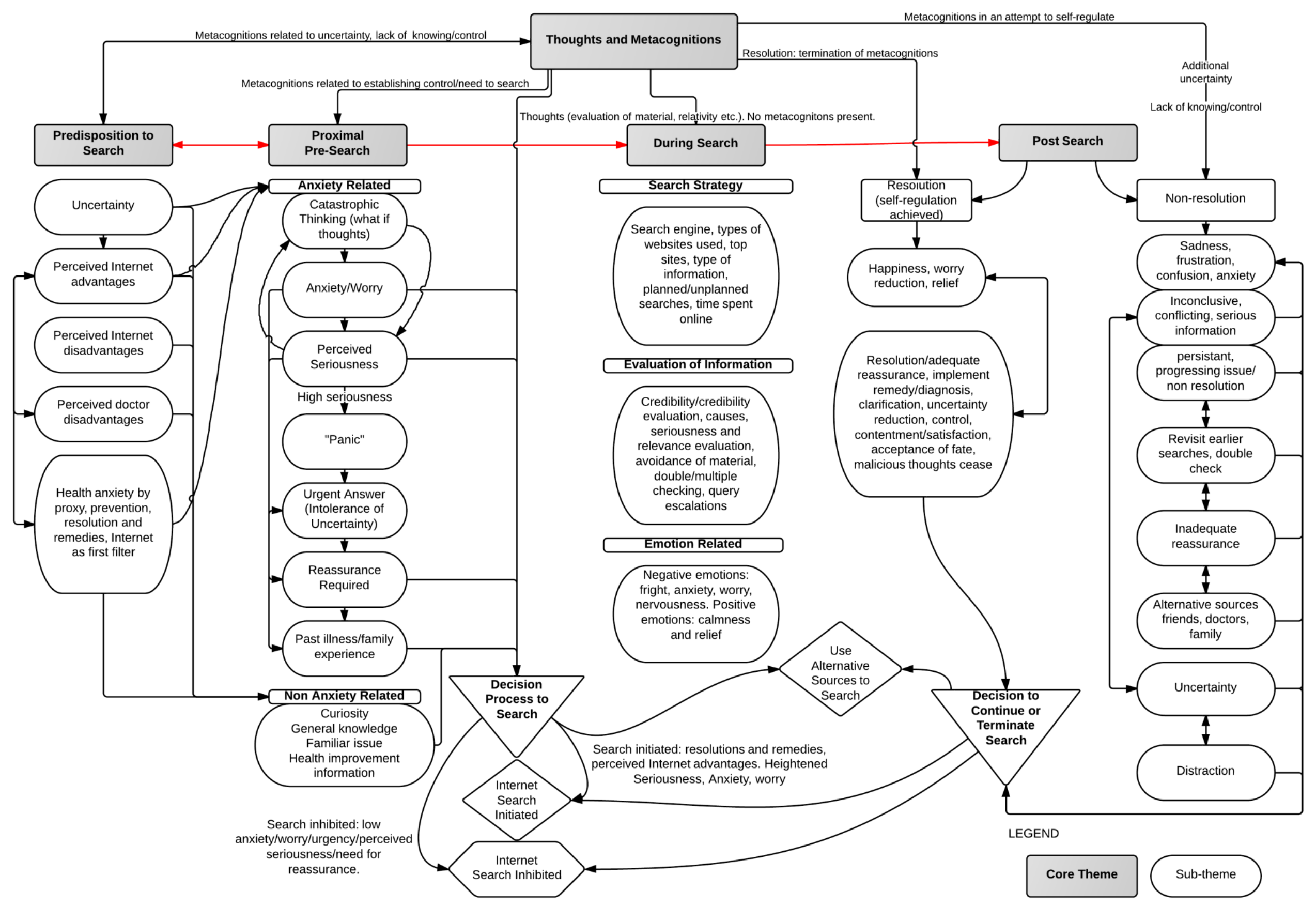

Figure 2. Summary of inter-intra theme relationships. 
Conversely, uncertainty about issues perceived as non-serious (e.g. those familiar to the individual such as acne) primed curiosity. Curiosity was a user response not underpinned by anxiety, and did not cause anxiety during or after the search. Curiosity-based searches used to obtain general knowledge and were not necessarily initiated with urgency.

Perceived advantages/disadvantages of the Internet. The majority of our participants used the Internet for health purposes because they perceived clear-cut advantages in doing so compared to alternative methods. Speed, convenience (including constant availability), ability to obtain opinions/advice, size and simplicity of the resource, and its ability to provide reassurance (as supported by successful searching in the past [reduced uncertainty]) were major incentives for health related Internet utilization. Users considered these advantages paramount in turning to the medium in order to obtain online health information. Participants suggested several Internet advantages were a direct result of being solutions to doctor disadvantages (see below).

“...It's very convenient to get information without actually going to the doctor. It [the Internet] answers you immediately. With the doctor I might get an answer in one or two days, with the Internet I get an answer within one or two hours..." (Participant 9)

However, a minority of our participants reported that their Internet-based reassurance was short lasting, which suggests that although the Internet was seen as a quick and resourceful medium, for some participants it was not one that could provide lasting cessation of anxiety and uncertainty. Participants also perceived several other disadvantages to the Internet, including it being too broad, confusing and providing too much information, being too vague, and providing conflicting information that heightened uncertainty and anxiety as it hindered the ability to come to a successful resolution (see post-search sub-theme below). These perceived disadvantages were often direct opposites of advantages (e.g. large resource/too broad), which suggests that 'too much of a good thing can be bad'. Relatedly, participants acknowledged the presence of false, non-credible, and/or irrelevant information, which increased skepticism of using the resource to obtain health information.

The majority of our participants also felt that the Internet contained alarming and anxiety provoking information, which increased disease conviction, was inconclusive, and planted negative thoughts (e.g. consequences of illness) in their minds. This suggests that the Internet is not considered a utopian solution to health problems by participants. It may be regarded as better than doctors, but not infallible in itself.

“...Sometimes it's like there's so many options, that you just get a bit lost and you think 'oh it can be any of these things'. So in that sense it might not necessarily be that helpful..." (Participant 2)

Perceived disadvantages of doctors. Participant responses suggested a clear link between perceived Internet advantages and doctor disadvantages. Several Internet advantages were direct solutions to doctor disadvantages. Most notably, distrust (including reluctance to prescribe medication or take one's concerns seriously), inconvenience, short appointment times, inadequate reassurance, and past negative experiences often set forward the motivation to use the Internet for health purposes. A minority of participants felt that these disadvantages/concerns were great enough to warrant doctor use only as a last resort. Responses suggested that the Internet would be used less if these doctor issues were reduced or resolved. However, no participant offered any advice on how such issues could be ameliorated.

"...It tends to be quite difficult to get appointments with the doctor and having to find a slot. Whereas this [the Internet] like you can go on at any time of day, and there's no time restriction on it. You can look at something for like hours and hours, whereas the doctor, you might only be able to talk to them for like 10 minutes..." (Participant 2)

Preventing IIIness. Preventing a serious future issue, and the deterioration of an existing issue, were noteworthy motivators for using the Internet in a majority of our sample. A connection between prevention and urgency was also uncovered. Participants utilized the Internet (due to its easy accessibility) to quickly resolve uncertainty about an issue. The prospect of not preventing a health problem was associated with the anticipation of dire consequences, furthering a sense of urgency, and the need to search online to resolve it. A lack of preventative measures often prompted anxiety amongst participants. Contrarily however, issues that 
were familiar to the participant (e.g. chronic symptoms, genetically predisposed symptoms, and symptoms already discussed with the doctor) were searched for in order to prevent acute/chronic worsening. In this sense, interview data further suggested a link between prevention and control, where participants proposed that actively searching the Internet to prevent an illness/genetic predisposition offered a sense of increased control.

“...My mother died of cancer, so it's deep in my maternal roots. I just feel like I'm predisposed. If I'm worrying about cancer and the Internet can provide a solution or possible explanation, it makes me worry a bit less..." (Participant 17)

Previous health experiences (in oneself or others) seemed to have a bearing on participants' decision to search in the majority of cases. Searches might be initiated in the proximal pre-search stage because participants wanted information about diagnoses given to them or others. Searches for others often followed a similar, but inhibited form of searching for one's own health. Suggesting concern and uncertainty regarding illness likelihoods for loved ones. It is noteworthy that key differences between searching for oneself and for others included guilt of not searching, sympathy prompting an Internet search, and the feeling of responsibility for a loved one's health. Whereas this was not present for one's own health. Preventing deterioration of diagnosed/undiagnosed illnesses was a theme for some. In these cases, curiosity and/or the desire to search for general information, rather than anxiety, was the major motivator to investigate in the proximal pre-search stage.

“... A lot of things, you don't feel them now, but in a few years they will come back. So I am also thinking about that; ways to start controlling the cholesterol..." (Participant 1)

Resolutions and remedies. Needing resolutions/remedies for pre-diagnosed/chronic and acute issues predisposed online searching for the majority of our participants. Depending on the perceived seriousness of the issue, these either led to anxious responses or curiosity in the proximal pre-search stage. Interview data also revealed a strong link between the need for resolution and anxiety. Moreover, unresolved issues promoted anxiety, requiring searches for resolutions/remedies to resolve them. The need for resolution/remedy both prompted initial searches and if no solutions were found, additional searches. When resolutions were found, searching was terminated; non-resolutions often led to additional searching (see below).

As with prevention, a feeling of control was present amongst participants when actively searching the Internet for resolutions or remedies, several participants suggested that simply using the Internet to find information was comforting in itself.

"...I just type in my symptoms to find out what I should do, and get concrete advice on how to act..." (Participant 4)

Internet as a first filter. Regarding the Internet as a "first filter" for health issues prior to doctor consultation was extremely popular in our sample. All of our participants said they used the Internet as a first source to filter out their health issues before moving to other resources such as their doctor. Reasons included reducing anxiety and uncertainty by understanding the issue, examining various possibilities, and avoiding embarrassment during appointments (e.g. to resolve sensitive issues [i.e. sexuality] anonymously). Using the Internet as a first filter also further supports our earlier findings with the relationship between Internet advantages and doctor disadvantages.

Relatedly, data also suggested many participants also used the Internet to gather information about possible diagnoses and engage in self-affirming experiments prior to a doctor visit. This was to both avoid wasting doctor time/resources, be better prepared for appointments (again to empirically "prove" disease existence to the doctor; justify the doctor visit), and be more efficient for the little time that was allocated to them during an appointment.

“...Well I don't think they [doctors] would believe you as much if you went in too often, because they would just consider you to be a hypochondriac. I think some doctors might just, even subconsciously, try to calm you down, whilst really, you might be going in with a genuine problem. You might appear less credible if you go all the time, that's why the Internet is much better in that 
way. The main thing about the Internet is that you can try to filter out things that aren't necessarily that serious..." (Participant 12)

\section{Proximal Pre-search}

In contrast to the more long-term characteristics and processes contained in the 'predisposition to search' theme, the proximal pre-search stage incorporates thoughts and characteristics present immediately before deciding to initiate or inhibit an Internet search. Participants' uncertainty/urgency and anxiety about their health status, coupled with their pre-existing beliefs, seemed to be key factors in this decision process.

The perceived seriousness of an issue, anxiety, and catastrophic thinking all contributed to decisions about whether to consult the Internet or alternative sources. Serious issues prompted sooner, more frequent and longer searches, seemingly to reduce uncertainty/anxiety, and obtain reassurance by finding answers. Searches were less likely, less urgent or shorter, if issues were perceived as trivial.

Non-anxiety related reasons for searching were also present in a minority of participants in the immediate presearch stage, including: curiosity, searching for general knowledge or health improvement information (e.g. exercise/diet), and searching about non-anxiety provoking existing/familiar issues. Searches in these areas did not cause anxiety before, during or after searches.

\section{During search}

The interviews revealed three main areas during health related web searches:

Search strategy. Interviews suggested Google was the primary resource used to initiate searching, likely due to its predominance as a search tool. Public professional resources (e.g., NHS websites) were preferred; however, private resources such as WebMD and personal blogs/forums were also popular. Illness familiarity and the type of information sought (general/preventative/remedy) often dictated which source was used. NHS or public/private equivalents were commonly used for unfamiliar illnesses; familiar or pre-diagnosed issues generally led towards blogs/forums and Wikipedia.

Time spent searching was proportionate to the issue's perceived seriousness: serious issues required longer search times than more trivial issues. Time searching also depended on interest, familiarity and complexity of the issue, and the participants' search skills. Planned searches (i.e., searches for pre-diagnosed/chronic issues, general information or health improvement information) were generally longer than unplanned searches (i.e., searches for unknown/acute issues and symptom information).

Some participants' descriptions suggested that they escalated their queries to searches for possibly serious causes for their symptoms (commonly due to uncovering unexpected and/or worrying information).

"...If you have a headache and you search for what could be causing that headache, obviously there are common things like you could just be tired. But then you'll end up seeing some sort of horrible thing like brain tumor, and then you'll go and check at what the other symptoms are for brain tumors to see if you've got that, and then you'll scare yourself so much that you've completely forgot you've got the headache in the first place..." (Participant 13)

Partial avoidance of online information was present in a minority of participants, particularly avoidance of alarming wording and other anxiety-inducing information. Conversely, a smaller minority of participants said they did not avoid anything online, suggesting avoidance was dependent on the participants' issue, level of health anxiety, and willingness to accept uncertainty.

A minority of participants utilized multiple/double checking, particularly after uncovering alarming or uncertain information, suggesting preoccupation with the health issue, and failure to attain sustained reassurance. 
Evaluation of information. The credibility of online information was important to the majority of participants. Several idiosyncratic search strategies were used to obtain and evaluate online health information. Non-serious issues required less credible sources (no supporting information, amateur layout etc.) such as personal blogs or community forums. Issues deemed serious required credible sources such as governmental or institutional websites. Techniques used to evaluate credibility online included: personal judgment/experience, utilizing academic websites, using advice/reviews from previous users, checking references/doctor credentials, and the professionalism of content (e.g., appropriate diction/grammar).

“...Even we [students] have to write things in a scientific way with short sentences and perfect grammar. On not very good websites sometimes there's bad punctuation; you can tell that it's written by someone who doesn't know what they're talking about. They're not even using proper words..." (Participant 3)

A minority of participants seemed to make intuitive judgments about credibility rather than formally critiquing websites.

“...By reading them, I get a feeling. I kind of find out if they are real or not..." (Participant 9)

Potential causes of symptoms, the likely seriousness of issues, and the relevance of online information were noteworthy areas that were evaluated during searches. Probable benign causes resulted in reassurance that, if enduring, led to acceptance and search termination. Casual information deemed irrelevant was often dismissed and avoided in subsequent searches. Contrastingly, web information that suggested serious issues/causes tended to intensify anxiety and prompted further reassurance seeking from the Internet or alternative sources (especially if the issues/causes seemed personally relevant).

“...I had bumps inside of my mouth and tried to find it [causes] on the Internet. I found this forum and it said that the bump was a kind of tumour. It described it exactly the same as with what I was actually having. After finding this [information] I could not concentrate anymore. I was like 'oh my God, what if it is a tumor'. Then I talked to my friend about it straight away..." (Participant 15)

Emotion related. Both negative (uncertainty, fright, anxiety, worry, and nervousness) and positive emotions (calm and relief) were present during searches. The emotion experienced was determined both by what was sought and what was found. Negative emotions were commonly associated with unfamiliar issues and calmness/neutrality/relief with familiar issues. Finding non-serious causes for symptoms prompted relief, whereas potentially alarming information incited fright and worry. The majority of our participants had found alarming information online, prompting additional searches. Contrastingly, non-alarming information resulted in search termination (please refer to post search theme below).

\section{Post Search}

Thoughts concerning the probable relevance and seriousness of the health information found online typically marked the transition into the post-search stage. Themes pertaining to the period following health related Internet use were grouped according to outcome: either resolution (search process ceases) or non-resolution (new search initiated, and/or alternative source consulted).

Resolution. When searches resulted in positive emotions (happiness, contentment, satisfaction, calmness, and relief) the search process was often terminated and further searches inhibited. Such feelings resulted from finding non-serious answers, diagnosing oneself, finding and/or implementing a remedy, successfully obtaining reassurance, and clarifying uncertainties. This also resulted in a sense of control, and reductions in both anxiety and illness thoughts/metacognitions.

“...When I had the exam period in my first semester I was extremely anxious; basically the whole left side of my body was kind of tingling all the time. So badly, that I was sure I was going to have a heart attack (laughs). So I wanted to check [online], and I saw a bunch of people saying the same thing. It 
just turned out that it will go away, like you just have to rest. So in that case yeah, it was reassuring..." (Participant 11)

Acceptance of an issue was also present for non-anxiety provoking matters with some participants setting aside queries because they had accepted their illness/diagnosis, fate, and thoughts.

“... I mean after you've put in sincere effort for 2-3 months [searching online], and you've taken medicines, and you are following the instructions given by the doctor. If you're not showing any considerable amount of changes, you know there's acceptance that you can't help anything...then you think okay fine let's leave it..." (Participant 19)

Non-resolution. Non-resolutions often resulted in negative emotions (sadness, frustration, confusion, and anxiety), and from information that was perceived as inconclusive, conflicting/confusing, and indicative of serious illness. Negative emotions were also associated with previously searched health issues persisting or progressing, and a lack of reassurance. In these cases, participants felt that the Internet had failed to resolve their uncertainty, anxiety, and negative illness thoughts. However, the Internet was still seen as the foremost source to obtain health information regardless. To overcome these Internet shortcomings, participants regularly consulted alternative and auxiliary sources such as friends, family, and doctors for advice/opinions regarding symptoms and information uncovered online. Often this was to obtain more reassurance (where the Internet had failed). Previous searches were also double/triple checked online, suggesting preoccupation. Nonresolutions encouraged fragmented searches over multiple sessions; it is unclear whether these were due to time constraints or due to information encountered online (i.e. lack of lasting reassurance), however. Participants with non-resolved queries also utilized distractions to forget about their issue and reduce uncertainty. These attempts usually proved futile, initiating renewed searching. In this case distraction may have been negatively reinforcing both anxiety and uncertainty.

"...If I feel really anxious about it, l'll go see my doctor and get checked properly. If I looked online and they [symptoms] continued to persist, then I might feel the need to [visit a doctor], even if it's just for my peace of mind..." (Participant 10)

\section{Metacognitions}

Participants described thought processes and metacognitions (i.e., thoughts about mental events themselves) related to before and after the search process, and the transition to the post-search stage. Therefore, metacognitions can be viewed as an overarching theme.

Before considering searching, participants attempted to weigh evidence supporting or mitigating the seriousness and likelihood of their health issue, in order to reassure themselves, to reduce anxiety/worry, uncertainty, and the perceived likelihood of having a serious illness. The most frequently cited metacognitions at this stage were negative beliefs related to uncertainty. The majority of participants were compelled to search online because they struggled with feelings of not knowing.

“...I'm not really sure myself. I just can't wait, I need the answers straight away or else I won't be able to think straight. I'm not going to be able to carry on [with daily routine]..." (Participant 15)

Negative metacognitions suggested problems with controlling/resisting Internet searches, despite being aware of Internet disadvantages. During this stage, thoughts about consequences and illness were linked with uncertainty, and evaluated further during a decision making phase. If pressing enough, these thoughts contributed to consulting the Internet/alternative sources.

“...I do it [search online] because I just need to know..." (Participant 11)

Both positive and negative metacognitions about "control" were also apparent in the proximal pre-search stage. Positive metacognitions concerned preventative searching in a minority of participants (e.g. "better to be safe 
than sorry"), which provided a sense of control over their fate, and helped to reduce anxiety and uncertainty. Furthermore, control also aided calmness, increasing the likelihood of a search being terminated.

“...I just sort of think of the worst case scenario. It's a way of preparing yourself; it's preparing me in case it is something bad. I feel as though if I prepare for the worst, then I'm prepared to deal with it. Whereas if I don't think about the worst, then I'm not prepared to meet the potential demands of it being something awful ..." (Participant 20)

In the during search stage, metacognitions about uncertainty and consequences were lowered because the focus was primarily on evaluating the possibilities, relevance, and likelihood of information encountered. Metacognitions in the post-search stage, related to additional uncertainty, anticipated consequences, and lack of control (if no resolution was found). Metacognitions were terminated with successful searches.

\section{Discussion}

We believe that this is one of the first published qualitative studies exploring the perspectives of health anxious individuals on their health related Internet use, and its impact upon them. Thematic analysis uncovered several core themes that could be organized chronologically. Findings were largely consistent with several concepts from the CBT theory/model of health anxiety (Warwick \& Salkovskis, 1990) but extend previous empirical research by demonstrating how concepts such as intolerance of uncertainty (Fergus, 2013, 2015; Norr et al., 2015), and the need for reassurance (Singh \& Brown, 2015) apply to health related Internet use. This study also demonstrates how theoretical concepts such as catastrophic thinking (Barsky, 2001), inflated likelihoods of illness presence and acquisition/hypervigilence (Starcevic, 2005), and dysfunctional illness beliefs apply to health related Internet use (theoretically; Marcus, Gurley, Marchi and Bauer, 2007, empirically; Fergus, 2013, 2015; Singh \& Brown, 2015). In particular, intolerance for uncertainty was an important finding in this study and is a sub-theme which extends across the search timeframe (i.e. predisposition to search, proximal pre-search, and post search; see figure 2). Previous studies (Fergus, 2013, 2015; Norr et al., 2015) have uncovered an empirical link between intolerance of uncertainty, health related Internet use, and distress. Our findings support these studies in demonstrating from a health anxious user's point of view, the importance of uncertainty in initiating a search (i.e. symptom-related uncertainty) and post-search (i.e. inconclusive findings, confusing information etc.).

Some findings were specific to an online context, including the perceived advantages/disadvantages of Internet use, the search strategy used, and how online information was evaluated in terms of relevance and credibility. Other findings could equally be explained using other models of health anxiety. For example, participants who experienced non-resolution of their queries often cited inadequate reassurance obtained online, which prompted the use of alternative sources (i.e. friends, family etc.) to obtain this reassurance. This finding may suggest the presence of attachment issues/insecurity, as suggested by the interpersonal model of health anxiety (Alberts \& Hadjistavropoulos, 2013). Similarly, these reassurance findings related to metacognitions could be explained using metacognitive theory (explained further below). Our results also support CBT theory, which posits that this lack of lasting reassurance is a major, hallmark, behavioral maintenance factor which serves to negatively reinforce preoccupation and dysfunctional illness beliefs (Creed \& Barsky, 2004; Starcevic, 2005).

Interview data revealed several perceived advantages in using the Internet for health purposes, this is a unique qualitative finding which supports earlier quantitative research in both health-anxious (Singh \& Brown, 2014) and non-health anxious (e.g. privacy for embarrassing issues; Gray et al., 2005) samples. According to our sample, often these perceived Internet advantages were direct reversals of doctor disadvantages, suggesting heightened doctor mistrust/perceived disadvantages in health anxious individuals (Singh \& Brown, 2014). Though both non/health anxious samples have reported perceived doctor disadvantages empirically, from a theoretical standpoint (Warwick \& Salkovskis, 1990; Wells, 1997) those with health anxiety may be more inclined towards experiencing/reporting doctor disadvantages. Perceiving these disadvantages might also lead to 'doctor shopping' or using alternative sources such as the Internet (Guo, Kuroki, Yamashiro, \& Koizumi, 2002; Norr et al., 2015). Consistent with this, interviews indicated that participants used the Internet to filter problems before consulting doctors, and to become 'expert patients'. This was another unique qualitative finding. Becoming an 
expert patient helped participants to 'sell' an illness to doctors, to gather information justifying a doctor visit, and to rapidly reduce anxiety and uncertainty before a doctor visit. This finding also supports CBT theory relating to doctor distrust and doctor shopping (Wells, 1997), where doctor distrust could lead to a need to justify one's concerns, and/or find alternative doctors who 'believe' that the patient is seriously ill. Furthermore, our finding could represent a form of self-empowerment using Internet-based health information (Korp, 2006), and/or further evidence of past negative doctor experiences.

In another unique qualitative finding, several participants recognized that doctors were irreplaceable due to their clinical intuition (i.e. doctor advantages), suggesting that in some cases doctor disadvantages related to time and convenience were the most likely to prompt Internet use, and not the content of appointments (Persing, Stuart, Noyes, \& Happel, 2000). This finding may also explain heightened post-search doctor utilization in high health anxious individuals (e.g., Baumgartner \& Hartmann, 2011; Eastin \& Guinsler, 2006; Muse et al., 2012; Singh \& Brown, 2014). These doctor disadvantages, paired with the Internet's ability to reassure and reduce anxiety/uncertainty was a major reason to use online health resources. Indeed, health anxious individuals experiencing an intolerance of uncertainty and a mistrust of medical professionals have been found to utilize online health resources more (Norr et al., 2015). Future research might consider whether resolving doctor disadvantages has any effect on perceived Internet advantages and utilization.

Interviews also suggested several disadvantages to using the Internet for health information, including containing too much, potentially conflicting, and alarming information. This was another unique qualitative finding. Internet utilization after recognizing disadvantages may be because benefits are perceived to outweigh costs. During searches, the use of personal skills and experiences were used to evaluate credibility, relevance and types of sources, perhaps as an attempt to ameliorate such disadvantages. This supports earlier findings (Singh \& Brown, 2015) suggesting evaluation of credibility is an important search behavior in health anxious individuals. However, it should be noted that due to their academic background, university students may have more knowledge about credible sources. Therefore, these evaluative skills may not be readily present in other (as demonstrated in older; Eysenbach and Kohler, 2002, and younger [e.g. middle school students]; Gray et al., 2005) samples.

Albeit, these skills have been demonstrated in research with non-health anxious samples (Eysenbach \& Kohler, 2002). Similar to findings by Eysenbach and Kohler's (2002) non health anxious sample, our sample used evaluative criteria such as content written by an official author, professional layout, professional writing style, and scientific references. This suggests that evaluation of credibility is deemed important for online health related searching regardless of state-trait health anxiety, and may constitute a more generalized safety-net behavior by users to evaluate health information online.

Participants described both positive and negative metacognitions about control. These findings were unique to our qualitative study. Positive metacognitions offered participants a sense of control in relation to their health. Prevention (of both novel and previously diagnosed problems) may represent an attempt to gain control over factors (e.g., uncertainty, catastrophising) that exacerbate health anxiety. This finding may also support earlier research suggesting heightened wellness-related searching in health anxious individuals (Singh \& Brown, 2014), as wellness information in these cases relates to disease prevention rather than treatment. This need for prevention, resolutions and reassurance was similar for the health of others, suggesting some participants experienced health anxiety by proxy. This was a unique qualitative finding.

Negative metacognitions were also described about uncertainty and loss of control over using the Internet for health purposes (e.g. not having the Internet available for health related searching). This may explain earlier research suggesting the presence of possible Internet addiction for health purposes in health anxious individuals (Singh \& Brown, 2014).

Time spent and emotions experienced whilst searching were dependent on the perceived seriousness and consequences of an issue, issue relevancy, issue familiarity/uncertainty, and the level of anxiety experienced. This is supported by Singh and Brown (2015) who found that perceived seriousness and issue unfamiliarity sometimes caused consideration of more serious health issues for one's symptoms (Singh \& Brown, 2015). Furthermore, negative emotions during searches were strongly associated with non-resolution of the search 
process, whereas positive emotions during searching were strongly linked to successful resolutions and search terminations, potentially leading to negatively reinforcing behaviour and the need for/lack of control in searching. This supports past research finding both relief (suggestive of successful resolution; Singh \& Brown, 2014), and distress/tension/anxiety (suggesting non-resolution; Baumgartner \& Hartmann, 2011; Muse et al., 2012; Singh \& Brown, 2014, 2015) in high health anxious individuals post-search. It also relates to the type of information searched for, as well as the perceived seriousness and familiarity of the issue. When seriousness and uncertainty were heightened, they required the use of more credible sources. These findings were unique to our qualitative methodology.

Resolutions and remedies offered a sense of control, reassurance, and reduced illness anxiety, uncertainty and preoccupation, and were therefore a major motivation to search. These are all areas of importance according to CBT theory of health anxiety (Starcevic \& Berle, 2013; Warwick \& Salkovskis, 1990; Wells, 1997). Therefore, suggesting that online health searching can reduce some of the distress associated with ambiguous symptoms. However, reassurance is theorized to be short lasting in health anxious individuals, leading to additional searches (Creed \& Barsky, 2004). Our finding of non-resolution prompting double/multiple searches after the initial search supports this idea, as non-resolutions maintained preoccupation arising from uncertainty and short-lasting reassurance. Relatedly, query escalation (i.e. searches of benign symptoms leading to considering serious causes; White \& Horvitz, 2009) in a subset of the sample and confusion deriving from conflicting information often led to the maintenance of illness thoughts and anxiety, leading to additional/revisited searches to resolve discrepancies, gain reassurance, and reduce anxiety. This finding supports earlier research which found increased anxiety following query escalations by health anxious individuals whilst searching for online health information (Singh \& Brown, 2015).

Avoidance and distraction were also present in several participants, which constitute potentially negatively reinforcing maintenance behaviours suggesting preoccupation (Hadjistavropoulos, Hadjistavropoulos, \& Quine, 2000; Olatunji, Deacon, \& Abramowitz, 2009). Finally, some participants said they stopped searching and accepted their symptoms/illness. Such acceptance usually occurred if the issue was familiar and not perceived as serious but might also occur due to frustration with the lack of a clear/feasible answer/information, or when the time spent searching was not justified by the perceived seriousness of the symptoms/illness. Usually acceptance related to searches for familiar issues, suggesting that preoccupation nor uncertainty were issues in these cases.

In conclusion, the current research suggests that health related Internet use has the potential of maintaining health anxiety in vulnerable (i.e. health anxious) individuals. The results generally support past theory in the idea that health related Internet use could act as a maintenance or initiatory factor for health anxiety. However, results also suggest that in some cases, the Internet can be beneficial in reducing health anxieties/concerns. It is currently unknown however, what specific online behaviour/information reduces or increases individual anxieties, clearly further research is required to examine these differences further.

Results suggested that anxiety might not always prompt a search (e.g. searches can be for general knowledge, curiosity), but may occur as a result of online health searching. This finding is supported by Singh and Brown (2015). This finding also underscores some of the potential hazards of using the Internet to obtain health information. However, feelings/emotions experienced post-search, and the resources and strategies employed during search, were largely dependent on the initial search purpose. Searches for acute/unknown ailments frequently led to post-search anxiety, whereas searches for familiar issues were largely reassuring. Similarly, the current study suggests that health anxious Internet users are not passively obtaining health information, but rather utilize a variety of strategies to obtain relevant and seemingly credible information. This supports the idea that health anxious individuals exercise caution in using the Internet, despite perceiving its advantages for health information; a finding also supported by Singh and Brown (2015).

Several limitations of the current study should be noted. Because the current study was conducted as part of a doctoral thesis the transcripts were coded and interpreted by only the first author. As such this study also lacks inter-coder agreement. However, data collection, transcription, coding, and analysis were subject to weekly supervisory checks from the second and third authors. It is also possible that our prior knowledge of CBT is likely to have influenced the presentation and interpretation of the data, despite making every effort to ensure the findings were data driven, with any interpretations relevant to theory coming after the fact. In this respect, pilot 
interviews and second/third author input at this piloting stage was used to minimize any potential bias before live data collection. Data were compiled into chronological stages to categorize and interpret themes in a manner similar to real world Internet usage for health information. As such, sub-themes were typically limited to one stage during interpretation in order to simplify presentation and interpretation of results. However, it is possible that themes omitted in one stage were present in others (e.g. avoidance may be present as a predisposing issue, during search, and post-searching). Moreover, inter-intra category relationships between themes are extremely complex, overlapping, and in many cases, idiosyncratic. Future research may further explore these relationships by examining links between specific themes in detail. Future research may also examine these themes further by observing our found areas of health related Internet searching in vivo, and qualitatively questioning its impact on the subject's health anxiety, similar to the study conducted by Singh and Brown (2015). Another limitation is that, although we believe theoretical saturation was achieved, the sample size of the study (20) is still relatively low and was restricted to university students with high self-reported health anxiety. As such, results may not be readily applicable to individuals with clinically significant levels of health anxiety. However, the mean health anxiety score of our sample (27.05) was higher than scores reported in several studies using non-clinical samples (Abramowitz, Deacon, \& Valentiner, 2007; 10.79; Roberts, Hart, \& Eastwood, 2009; 13.38), albeit slightly lower than studies with clinical samples (Abramowitz \& Moore, 2007; 33.3, Deacon \& Abramowitz, 2008; 31.22). Replication with a larger, preferably clinical, sample is clearly required. Similarly, it would be useful to repeat the study in a cohort with different demographics, to determine any disparities in health related Internet use due to psychosocial diversity, technological experience, search skills etc.

Despite these limitations the current study provides useful clinical information by reporting the prominent health searching related thoughts and behaviours of high health anxious individuals, which might inform both preventative and curative interventions. Similarly, these findings might be used by researchers to develop or refine quantitative Internet-use measures to better determine areas of importance related to online health information searching in high health anxious individuals.

\section{References}

Abramowitz, J. S., Deacon, B. J., \& Valentiner, D. P. (2007). The short health anxiety inventory: psychometric properties and construct validity in a non-clinical sample. Cognitive Therapy and Research, 31, 871-883. http://dx.doi.org/10.1007/s10608-006-9058-1

Abramowitz, J. S., \& Moore, E. L. (2007). An experimental analysis of hypochondriasis. Behaviour Research and Therapy, 45, 413-424. http://dx.doi.org/10.1016/j.brat.2006.04.005

Abramowitz, J. S., Olatunji, B. O., \& Deacon, B. J. (2007). Health anxiety, hypochondriasis, and the anxiety disorders. Behavior Therapy, 38, 86-94.

Alberts, N. M., \& Hadjistavropoulos, H. D. (2013). Parental illness, attachment dimensions, and health beliefs: testing the cognitive-behavioural and interpersonal models of health anxiety. Anxiety, Stress \& Coping. Advanced online publication. http://dx.doi.org/10.1080/10615806.2013.835401

Alberts, N. M., Hadjistavropoulos, H. D., Jones, S. L., \& Sharpe, D. (2013). The short health anxiety inventory: a systematic review and meta-analysis. Journal of Anxiety Disorders, 27, 68-78.

http://dx.doi.org/10.1016/j.janxdis.2012.10.009

Barsky, A. J. (2001). The patient with hypochondriasis. New England Journal of Medicine, 345, 1395-1399.

Baumgartner, S. E., \& Hartmann, T. (2011). The role of health anxiety in online health information search. Cyberpsychology, Behavior, and Social Networking, 14, 613-618. http://dx.doi.org/10.1089/cyber.2010.0425

Braun, V., \& Clarke, V. (2006). Using thematic analysis in psychology. Qualitative Research in Psychology, 3, 77-101. 
Conroy, R. M., Smyth, O., Siriwardena, R., \& Fernandes, P. (1999). Health anxiety and characteristics of selfinitiated general practitioner consultations. Journal of psychosomatic research, 46(1), 45-50.

Corbin, J., \& Strauss, A. (2014). Basics of qualitative research: Techniques and procedures for developing grounded theory. Sage publications.

Creed, F., \& Barsky, A. (2004). A systematic review of the epidemiology of somatization disorder and hypochondriasis. Journal of Psychosomatic Research, 56, 391-408. http://dx.doi.org/10.1016/S0022-3999(03)006226

Deacon, B., \& Abramowitz, J. S. (2008). Is hypochondriasis related to obsessive compulsive disorder, panic disorder, or both? An empirical evaluation. Journal of Cognitive Psychotherapy, 22, 115-127.

Eastin, M. S., \& Guinsler, N. M. (2006). Worried and wired: effects of health anxiety on information-seeking and health care utilization behaviors. CyberPsychology \& Behavior, 9, 494-498.

http://dx.doi.org/10.1089/cpb.2006.9.494

EU Commission. (2013). Managing health data. Retrieved from http://ec.europa.eu/digital-agenda/en/managinghealth-data

Eysenbach, G., \& Kohler, C. (2002). How do consumers search for and appraise health information on the world wide web? Qualitative study using focus groups, usability tests, and in-depth interviews. BMJ, 324, 573-577. http://dx.doi.org/10.1136/bmj.324.7337.573

Fergus, T. A. (2015). Anxiety sensitivity and intolerance of uncertainty as potential risk factors for cyberchondria: A replication and extension examining dimensions of each construct. Journal of affective disorders, 184, 305-309.

Fergus, T. A. (2013). Cyberchondria and intolerance of uncertainty: examining when individuals experience health anxiety in response to Internet searches for medical information. Cyberpsychology, Behavior, and Social Networking, 16, 735-739.

Fox, S. (2013). Pew Internet: Health. Retrieved from

http://www.pewInternet.org/Commentary/2011/November/Pew-Internet-Health.aspx

Gray, N. J., Klien, J. D., Noyce, P. R., Sesselberg, T. S., \& Cantrill, J. A. (2005). Health information-seeking behaviour in adolescence: the place of the Internet. Social Science \& Medicine, 60, 1467-1478.

http://dx.doi.org/10.1016/j.socscimed.2004.08.010

Guo, Y., Kuroki, T., Yamashiro, S., \& Koizumi, S. (2002). Illness behaviour and patient satisfaction as correlates of self-referral in japan. Family Practice, 19, 326-332. http://dx.doi.org/10.1093/fampra/19.4.326

Hadjistavropoulos, H. D., Craig, K. D., \& Hadjistavropoulos, T. (1998). Cognitive and behavioral responses to illness information: the role of health anxiety. Behaviour Research and Therapy, 36, 149-164.

Hadjistavropoulos, H. D., Hadjistavropoulos, T., \& Quine, A. (2000). Health anxiety moderates the effects of distraction versus attention to pain. Behaviour Research and Therapy, 38, 425-438.

Korp, P. (2006). Health on the Internet: implications for health promotion. Health Education Research, 21, 78-86.

Kummervold, P. E., Chronaki, C. E., Lausen, B., Prokosch, H. U., Rasmussen, J., Santana, S., Staniszewski, A., \& Wangberg, S. C. (2008). eHealth trends in Europe 2005-2007: A population-based survey. Journal of Medical Internet Research, 10(4).

Latthe, M., Latthe, P. M., \& Charlton, R. (2000). Quality of information on emergency contraception on the Internet, British Journal of Family Planning, 26(1), 39-43. 
Marcus, D. K., \& Church, S. E. (2003). Are dysfunctional beliefs about illness unique to hypochondriasis? Journal of Psychosomatic Research, 54, 543-547. http://dx.doi.org/10.1016/S0022-3999(02)00526-3

Marcus, D. K., Gurley, J. R., Marchi, M. M., \& Bauer, C. (2007). Cognitive and perceptual variables in hypochondriasis and health anxiety: A systematic review. Clinical Psychology Review, 27, 127-139.

Muse, K., McManus, F., Leung, B., Meghreblian, T., \& Williams, J. M. G. (2012). Cyberchondriasis: Fact or fiction? A preliminary examination of the relationship between health anxiety and searching for health information on the Internet. Journal of Anxiety Disorders, 26, 189-196. http://dx.doi.org/10.1016/j.janxdis.2011.11.005

NHS national health service: Improving access to psychological therapies (IAPT), (2011). The iapt data handbook. Retrieved from http://www.iapt.nhs.uk/silo/files/iapt-data-handbook-v2.pdf

Norr, A. M., Albanese, B. J., Oglesby, M. E., Allan, N. P., \& Schmidt, N. B. (2015). Anxiety sensitivity and intolerance of uncertainty as potential risk factors for cyberchondria. Journal of Affective Disorders, 174, 64-69.

Olatunji, B. O., Deacon, B. J., \& Abramowitz, J. S. (2009). Is hypochondriasis an anxiety disorder? The British Journal of Psychiatry, 194, 481-482.

Persing, J. S., Stuart, S. P., Noyes, R., \& Happel, R. L. (2000). Hypochondriasis: The patient's perspective. International Journal of Psychiatry in Medicine, 30, 329-342.

Roberts, K. E., Hart, T. A., \& Eastwood, J. D. (2009). Attentional biases to social and health threat words in individuals with and without high social anxiety or depression. Cognitive Therapy and Research, 34, 388-399.

Salkovskis, P. M., Rimes, K. A., Warwick, H. M. C., \& Clark, D. M. (2002). The health anxiety inventory: development and validation of scales for the measurement of health anxiety and hypochondriasis. Psychological Medicine, 32, 843-853. http://dx.doi.org/10.1017/S0033291702005822

Singh, K., \& Brown, R. J. (2014). Health related Internet habits and health anxiety in university students. Anxiety, Stress \& Coping, 27, 542-554. http://dx.doi.org/10.1080/10615806.2014.888061

Singh, K., \& Brown, R. J., (2015). From headache to tumour: An examination of health anxiety, health related Internet use, and 'query escalation'. Journal of Health Psychology. Advanced online publication.

Smith, J. A. (1996). Beyond the divide between cognition and discourse: Using interpretative phenomenological analysis in health psychology. Psychology and health, 11, 261-271.

Starcevic, V. (2005). Fear of death in hypochondriasis: Bodily threat and its treatment implications. Journal of Contemporary Psychotherapy, 35, 227-237.

Starcevic, V., \& Berle, D. (2013). Cyberchondria: Towards a better understanding of excessive health related Internet use. Expert Review of Neurotherapeutics, 13, 205-213.

von Scheele, C., Nordgren, L., Kempi, V., Hetta, J., \& Hallborg, A. (2010). A study of so-called hypochondriasis. Psychotherapy and Psychosomatics, 54, 50-56.

Warwick, H., \& Salkovskis, P. M. (1990). Hypochondriasis. Behaviour Research and Therapy, 28, 105-117.

Warwick, H. M., Clark, D. M., Cobb, A. M., \& Salkovskis, P. M. (1996). A controlled trial of cognitive-behavioural treatment of hypochondriasis. The British Journal of Psychiatry, 169, 189-195.

Warwick, H. M., \& Marks, I. M. (1988). Behavioural treatment of illness phobia and hypochondriasis. A pilot study of 17 cases. The British Journal of Psychiatry, 152, 239-241. 
Wells, A. (1997). Cognitive therapy of anxiety disorders: a practice manual and conceptual guide. Chichester, UK: John Wiley \& Sons Ltd.

White, R. W., \& Horvitz, E. (2009). Cyberchondria: Studies of the escalation of medical concerns in web search. ACM Transactions on Information Systems, 27(4), 1-37. Retrieved from http://research.microsoft.com/en us/um/people/ryenw/papers/WhiteTOIS2009.pdf

\section{Correspondence to:}

Karmpaul Singh

School of Psychological Sciences

University of Manchester

2nd Floor, Zochonis Building

Brunswick Street,

Manchester, U.K. M13 9PL

Email: karmpaul(at)gmail.com

\section{Appendix: Interview Guide}

Please note the following are some examples of questions we may use. They do not form a finite list of the questions we may ask, some questions will be guided by participant's responses.

The overarching goal of the study is to get a better understanding of health anxious individuals' experience of the Internet in relation to health and illness. More specifically, we hope to get an understanding of what, why and how health/illness information on the Internet is accessed by health anxious individuals and any effects that this has on what they think, feel and do. We hope to understand (questions in italics):

\section{Whether people end up on health/illness related websites}

Do you ever use the Internet for health purposes?

Possible pointers for follow up:

Can you tell me a little bit about that?

Is it something you do often?

How often?

It sounds a little like your searches for health information are planned/not planned, is this correct? Can you expand on that a little bit?

\section{What prompts people to access health/illness related websites and their reasons for doing so}

If you think back to a recent time when you used the Internet for health purposes, what motivated you to use it?

Possible pointers to follow up:

What was your goal when searching online at the time?

Do you often find your goal hard to achieve?

Can you talk a little bit about that? Why is it hard/ why isn't it hard?

The kinds of health/illness related websites that people access 
Can you tell me a little bit about how you actually get the information you want, such as any particular

preference/method in how you get the information you're looking for?

Possible pointers for follow up:

When you say you prefer using Google, that's in essence, just a search engine and a method of finding information, what sort of sites lead from that Google search?

Have you ever considered an alternative method?

What about this method works for you? What doesn't work?

\section{How they perceive these websites}

Can you tell me a little about how you feel about health websites themselves?

Possible pointers for follow-up:

What are some disadvantages or advantages that you perceive in these health websites?

Sounds like you perceive more advantages than disadvantages [vice versa], is this correct?

What is your opinion on these websites, do you find them beneficial often or rarely?

How important is the credibility of the information you search for? Can you talk a little about that?

\section{The kinds of information that people seek when using the Internet for health/illness related purposes}

Can you talk a little bit about the types of things you are looking for when you're searching for health purposes?

Possible pointers for follow:

Do you ever search for (other type of information health/illness)?

How often to you search for health/illness information?

Is there any particular order you search this information in?

Have you ever found yourself looking for information you originally weren't looking for? (query escalation) Can you talk a little bit about that?

Do you ever find alarming health information when you are online? What do you do when you find this alarming information online?

\section{The kinds of information that people obtain when using the Internet for health/illness related purposes.}

You mentioned you search for (type of information) quite often, do you usually find what you're looking for?

Possible pointers for follow up:

What are some of the other types of information you've stumbled upon that you had been unexpected?

Do you feel satisfied with what you find? What about it makes you satisfied/dissatisfied?

Have you ever been surprised with what you find?

\section{How people use health/illness related websites and what they do when they're on them}

Can you tell me a little about what you usually do when you're on these websites, just sort of walk me through it?

Possible Pointers for follow up:

Is there any particular strategy you use once you're on the website? For example do you try to focus on images on a website or prefer a text description?

What about this strategy works for you? What doesn't?

How long would you say it takes you to find the answer you're looking for on each website?

\section{What people think and feel when they're on a health/illness related website}

When you're actually on the website, while you are searching, what are some of the feelings you're having? 
Possible pointers for follow up:

Can you think of a recent time that you searched for health information and tell me what sort of things were going through your mind?

Can you expand a bit more on what thoughts are occurring?

How long do these feelings and thoughts last? What makes them stay/go away?

\section{What people think and feel after they've accessed health/illness related websites.}

In general what kind of affect does using the Internet for health purposes have on you emotionally and behaviorally?

Possible pointers for follow up:

So it sounds like you feel better/worse after you search, can you talk a little bit about that?

How long would you say these thoughts last? What about the feelings?

What sort of steps do you take to deal with these feelings?

How would you feel if the Internet was completely taken away for you for health purposes? (Addiction).

\section{What people do after they've accessed health and illness related websites}

So after you're done searching, what comes next?

Possible pointers for follow up:

Are you satisfied with what you find?

Is there anything you like to do or follow up on after searching for health related information online?

Do you consult a doctor after your search? Can you talk a little bit about that?

Let's pretend you're sitting here and you find a symptom that's bothering you, can you walk me through the typical procedure you may take to deal with that issue?

Possible pointers for follow-up:

Your general mindset at the time

What you would do?

If you go online what sort of things your aiming to see/find?

How you feel afterwards?

Addressing limitation from first study: Can you take some time and tell me what other issues are important to you when using the Internet for health purpose, something we may have missed or something you think that is important?

General probe questions (questions used to elicit further information from the participants):

Could you tell me a bit more about that?

This is what l've understood, is that right?

When you say. what do you mean? Could you give me an example? 


\begin{abstract}
About authors
Dr. Karmpaul Singh is a postdoctoral research fellow at the Centre for Applications of Health Psychology at the University of Southampton (UK). His research interests primarily relate to health anxiety and its relation to online contexts, and more broadly in e-health and 'cyber-psychology'. His past and current research interests have aimed to examine how technology is related to health behaviours. He is currently working on determining how such technology can be harnessed to help resolve detrimental instances of mental health, and improve quality of life for cancer survivors via online interventions.
\end{abstract}

Dr. John Fox is a consultant Clinical Psychologist, lecturer in clinical psychology (honorary), and an author/researcher in the field of mental health. He has published over 50 research studies and one book on eating disorders. His work has focused on emotional processing within eating disorders, psychological approaches to physical health (e.g. cancer, Tourette's syndrome), and qualitative research methodology.

Dr. Richard J Brown is Senior Lecturer in Clinical Psychology and Programme Director for the Clinical Psychology Doctorate at the University of Manchester. He is also Honorary Consultant Clinical Psychologist with Manchester Mental Health and Social Care NHS Trust, where he runs the Functional Neurological and Dissociative Disorders (FuNDD) Service. He has written numerous theoretical, empirical and clinical papers on "medically unexplained" symptoms, with a particular focus on the psychology of dissociation and functional neurological symptoms. He won the British Psychological Society May Davidson Award in 2012 for his work in this area. 